\title{
Conservação do morango armazenado em atmosfera modificada
}

\section{Strawberries storage conservation in modified atmosphere}

\author{
Rogério Lopes Vieites ${ }^{1 *}$; Regina Marta Evangelista ${ }^{1}$; \\ Cíntia de Souza Silva ${ }^{1}$; Marcelo Luis Martins ${ }^{1}$
}

\section{Resumo}

\begin{abstract}
Este trabalho teve como objetivo avaliar a qualidade e a conservação de morangos armazenados sob atmosfera modificada. Foram utilizados morangos 'Oso Grande', produzidos na cidade de Botucatu-SP e colhidos no estádio de maturação $3 / 4$ vermelho. O experimento constituiu no armazenamento das frutas em embalagens de polietileno sob diferentes misturas gasosas: testemunha; vácuo parcial; $5 \% \mathrm{O}_{2}$ e $1 \%$ $\mathrm{CO}_{2} ; 5 \% \mathrm{O}_{2}$ e $3 \% \mathrm{CO}_{2} ; 5 \% \mathrm{O}_{2}$ e $5 \% \mathrm{CO}_{2}$. Os parâmetros analisados foram: aparência, vida útil, incidência de doenças, teor de sólidos solúveis, acidez titulável, vitamina $\mathrm{C}$ e firmeza.. Para a avaliação da conservação pós-colheita, os frutos foram armazenados a $0^{\circ} \mathrm{C}+/-1^{\circ} \mathrm{C} / 90+/-5 \%$ UR por 12 dias e avaliados a cada dois dias. $\mathrm{O}$ delineamento estatístico adotado foi o inteiramente casualizado, comparando as médias pelo teste de Tukey a $5 \%$ de probabilidade. Os resultados obtidos permitem concluir que as frutas do tratamento testemunha não apresentaram diferenças estatísticas dos demais tratamentos exceto o de vácuo parcial. Palavras-chave: Fragaria ananassa Duch, mistura gasosa, armazenamento, pós-colheita
\end{abstract}

\begin{abstract}
Abctract
The objective of this work was to evaluate the quality and conservation of strawberries in modified atmosphere. 'Oso Grande' strawberries, produced in Botucatu-SP-Brazil, and picked in the $3 / 4$ red maturation stages were used. The experiment consisted in the storage of the fruits in polyethylene packages under different gaseous mixtures: control; vacuous; $5 \% \mathrm{O}_{2}$ and $1 \% \mathrm{CO}_{2} ; 5 \% \mathrm{O}_{2}$ and $3 \% \mathrm{CO}_{2} ; 5 \% \mathrm{O}_{2}$ and $5 \% \mathrm{CO}_{2}$. The analyzed parameters were: appearance, shelf life, incidence of disease, soluble solids, titratable acidity, vitamin $\mathrm{C}$ and firmness. For evaluation of the post-harvest conservation the fruits were stored in $0^{\circ} \mathrm{C}+/-1^{\circ} \mathrm{C} / 90+/-5 \%$ UR ambient for 12 days and appraised every 2 days. The statistical layout was completely randomized, by comparing the means with Tukey test in a ratio of $5 \%$ probability. The obtained results allow to conclude that the fruits of the treatment testify didn't present statistical differences of the other treatments except the one of vacuous partial.
\end{abstract}

Key words: Fragaria ananassa Duch, gaseous mixtures, storage, post harvest

1 Universidade Estadual Paulista (UNESP), Faculdade de Ciências Agronômicas, Departamento de Gestão e tecnologia Agroindustrial, Botucatu, SP. Email vieites@fca.unesp.br

* Autor para correspondência. 


\section{Introdução}

Os atributos de qualidade normalmente exigidos pelo consumidor para a maioria das frutas e hortaliças em geral são: aparência, sabor, odor, valor nutritivo, ausência de defeitos. No caso do morango, esta condição é facilmente atingida, já que esse fruto apresenta atração peculiar, por sua cor vermelhobrilhante, odor envolvente, textura macia e sabor levemente acidificado (SILVA, 2004).

Levando-se em consideração a aparência do morango armazenado em atmosfera modificada e sob refrigeração, Collins e Perkins-Veazie (1993) observaram que a queda na qualidade visual dos frutos durante o armazenamento foi pequena em comparação com os frutos armazenados em ambiente atmosférico normal. Os mesmos autores verificaram que a refrigeração foi vital para assegurar a manutenção da aparência. Segundo estes mesmo autores os frutos e hortaliças, como órgãos vivos, continuam a respirar após a colheita. Sendo assim, métodos que proporcionem baixas concentrações de oxigênio e altas concentrações de dióxido de carbono são hoje uma realidade no armazenamento de frutos e hortaliças, para a manutenção da qualidade e por estender a vida pós-colheita dos mesmos. Entre esses métodos se destaca a atmosfera modificada.

Chitarra e Chitarra (1990) citam que o período máximo de vida pós-colheita para o morango (10 dias) só é atingido se os frutos forem mantidos em temperaturas de $0-5^{\circ} \mathrm{C}, 10 \% \mathrm{O}_{2}$ e $15-20 \% \mathrm{CO}_{2}$.

Uma vez que a composição final da atmosfera não é controlada, mas é dependente da interação entre o produto, barreira e ambiente, o termo "atmosfera modificada" foi adotado para distinguir esta técnica da convencional atmosfera controlada (KADER, 1992).

Segundo Cia (2002), a habilidade para regular a atmosfera estabelecida na embalagem dependerá da respiração do fruto e da permeabilidade da embalagem. Esses fatores, por sua vez, são dependentes da temperatura, já que a elevação da mesma promove aumento da taxa respiratória dos produtos e da permeabilidade do filme utilizado. Portanto, o uso de embalagens não irá reduzir nem tão pouco eliminar a necessidade de refrigeração (SARANTÓPOULOS et al, 1996).

De acordo com Kluge et al (2001), níveis demasiadamente baixos de $\mathrm{O}_{2}$ levam os frutos a respiração anaeróbica, resultando em formação de acetaldeído e etanol, modificando o sabor e escurecendo os tecidos dos frutos. Já distúrbios causados pelo excesso de $\mathrm{CO}_{2}$ se caracterizam pelo amarronzeamento dos tecidos e formação de etanol.

O morango apresenta alta taxa respiratória após a sua colheita. Segundo Kader et al (1989) os materiais mais adequados usados como embalagens para produtos que ‘respiram' são filmes poliolefínicos (simples, laminados ou co-extrusados), devido às boas propriedades de barreira ao vapor d'água, relativamente alta taxa de permeabilidade $\mathrm{a}_{2} \mathrm{e} \mathrm{CO}_{2}$ e boas características de termosselagem. Formulações de cloreto de polivinila (PVC) também são utilizados, os quais apresentam moderada taxa de permeabilidade ao vapor d'água e podem apresentar altas taxas de permeabilidade ao $\mathrm{O}_{2}$ e $\mathrm{CO}_{2}$.

Vários trabalhos de pesquisa tem comprovado a eficiência do uso de atmosfera modificada em diversos frutos, provando o efetivo aumento do período do seu armazenamento, através da manutenção de seus atributos de qualidade (aparência, textura, sabor, odor e menor incidência de doenças).

Cia (2002) cita que com a utilização de filmes no acondicionamento dos frutos, verifica-se melhor apresentação do produto, eliminação ou redução do uso de fungicidas, redução da superfície de abrasão, diminuição do contato do fruto na sua comercialização, diminuição da contaminação do fruto durante o manuseio, manutenção de alta umidade relativa e redução da perda de massa. O uso da atmosfera modificada também reduz a incidência de deteriorações e podridões em morangos (FLORES-CANTILLANO, 1998). 
Hertog et al (1999), Silva (2002) e Van der Steen et al (2002) verificaram que as elevadas concentrações de $\mathrm{CO}_{2}$ no armazenamento de morangos foram eficientes na redução de perdas causadas por patógenos, principalmente por Botrytis cineria.

Scalon et al (1996) verificaram que a conservação de morangos a $4^{\circ} \mathrm{C}$ em embalagem de $\mathrm{PVC}$ prolongou a vida útil dos frutos para 14 dias, mantendo sua qualidade, uma vez que os teores de $\mathrm{pH}$, sólidos solúveis e acidez titulável, açúcar total e redutor encontravam-se na faixa ideal para consumo.

Berbari et al (1994) e Gil et al (1997) trabalhando com morangos armazenados com atmosferas modificadas sob diferentes concentrações de $\mathrm{CO}_{2}$, verificaram que os teores de sólidos solúveis diminuíram depois de 5 e 10 dias de armazenamento a $5^{\circ} \mathrm{C}$.

Chitarra e Chitarra (1990), Sigrist (1992) e Scalon (1996) citam que os teores de acidez titulável dos frutos tendem a cair durante o seu armazenamento.

Segundo Flores-Cantillano (1998), a análise conjunta de muitas variáveis permite observar que a qualidade sensorial e a acidez são parâmetros mais importantes na qualidade global dos morangos e que, principalmente os filmes poliméricos e as atmosferas com alto teor de $\mathrm{CO}_{2}$ contribuem para preservar a sua qualidade.

Nunes et al (1998) e Calegaro et al (2002) verificaram diminuição nos teores de vitamina $\mathrm{Cem}$ morango durante o seu armazenamento. Segundo Islan et al (1993) essa redução se dá devido a alta atividade pós-colheita da enzima ácido ascórbico oxidase. Para Gayon (1972) a diminuição dos teores de vitamina $\mathrm{C}$ durante a pós-colheita pode ser devido à menor capacidade dos vegetais sintetizar esse ácido durante este período.

Brackmann et al (2001), estudando os efeitos de armazenamento de morangos cv. Oso Grande sob elevadas pressões parciais de $\mathrm{CO}_{2}$, observaram que o uso de $20 \% \mathrm{CO}_{2}$ e baixa temperatura $\left(0^{\circ} \mathrm{C}\right)$ durante o armazenamento, transporte e comercialização propiciaram boa manutenção de qualidade por até
20 dias, não sendo verificado alterações no sabor e aroma das frutas.

Calegaro et al (2002) verificaram os benefícios decorrentes da utilização de atmosferas iniciais com $3 \% \mathrm{O}_{2}+10 \% \mathrm{CO}_{2}$ ou $5 \% \mathrm{O} 2+15 \% \mathrm{CO}_{2}$, em morangos cv. Oso Grande armazenados a $0^{\circ} \mathrm{C}$, que foram a manutenção da firmeza da polpa, da coloração e dos teores de açúcares totais e de ácido ascórbico.

De acordo com Santos (1999), a firmeza da polpa e a resistência da epiderme são características de extrema importância, especialmente para as cultivares destinadas à produção de fruto para o consumo in natura, pois além de permitirem melhor manuseio e transporte, possibilitam a conservação das qualidades sensoriais por mais tempo.

No decorrer do período de armazenamento de morangos em atmosferas modificadas, há um acúmulo de $\mathrm{CO}_{2}$, com queda na concentração de $\mathrm{O}_{2}$ (KAYS, 1991, NUNES et al, 1998 E VAN DER STEEN et al, 2002).

Estudos realizados com morangos verificaram que quando a fruta é armazenada sob altas concentrações de $\mathrm{CO}_{2}$, mantém a sua firmeza por mais tempo (Harris e Harvey, 1973, 1978, Mitchell, 1992). Li e Kader (1989) observaram que morangos armazenados por até 4 dias com $20 \%$ de $\mathrm{CO}_{2}$, mantiveram sua firmeza por maior período de tempo do que as frutas tratadas durante apenas 2 dias.

Harker et al (2000) em experimentos com morangos armazenados em elevadas concentrações de $\mathrm{CO}_{2}$ verificaram aumento de firmeza do morango em função da elevação da concentração de $\mathrm{CO}_{2}$ no ambiente de armazenamento. Holcroft e Kader (1999) relatam que a atmosfera com elevada concentração de $\mathrm{CO}_{2}$ foi eficiente no controle e retenção da firmeza de morangos armazenados durante 10 dias a $5^{\circ} \mathrm{C}$, enquanto que as frutas armazenadas em condições atmosféricas normais, amoleceram muito mais rápido nas mesmas condições de temperatura. 
Este trabalho teve como objetivo avaliar qualidade e a conservação do morango armazenado em diferentes condições de atmosfera modificada.

\section{Material e Métodos}

Foram utilizados para a pesquisa, morangos, cultivar "Oso Grande", no estádio de maturação 3/4 vermelho, produzidos na cidade de Botucatu-SP. Os frutos foram colhidos manualmente cortando-se o pedúnculo, a aproximadamente $1,0 \mathrm{~cm}$ acima do cálice. Depois de colhidos, foram levados para o Laboratório de Frutas e Hortaliças do Departamento de Gestão e Tecnologia Agroindustrial onde foram selecionados, visando a homogenização do lote.

Em seguida, foram submetidos à desinfecção, com solução de $200 \mathrm{mg} \mathrm{L}^{-1}$ de hipoclorito de sódio, por 2 minutos, à temperatura ambiente e secos ao ar.

Os frutos foram acondicionados em embalagem de polietileno transparente, denominado 'Nylon Poli', tendo como características a alta barreira ao oxigênio $\left(10 \mathrm{~cm}^{3} \mathrm{dia}^{-1}\right)$ e ao vapor d'água $\left(5 \mathrm{~m}^{2} \mathrm{dia}^{-1}\right)$. No fechamento, as embalagens foram submetidas a diferentes concentrações de $\mathrm{O}_{2}$ e $\mathrm{CO}_{2}$; a testemunha foi representada por frutos embalados com ar ambiente. $\mathrm{O}$ equipamento utilizado para a injeção dos gases e fechamento das embalagens foi uma "seladora a vácuo - TEC MAC AP 500".

As diferentes concentrações de $\mathrm{O}_{2}$ e $\mathrm{CO}_{2}$ constituíram os tratamentos: T1- Testemunha (ar atmosférico ambiente); T2 - Vácuo Parcial (vácuo até a não deformação dos frutos); T3 - 5\% $\mathrm{O}_{2}$ e $1 \%$ $\mathrm{CO}_{2} ; \mathrm{T} 4-5 \% \mathrm{O}_{2}$ e $3 \% \mathrm{CO}_{2}$ e $\mathrm{T} 5-5 \% \mathrm{O}_{2}$ e $5 \% \mathrm{CO}_{2}$.

Os frutos embalados foram armazenados em câmara fria a $0^{\circ} \mathrm{C}+/-2^{\circ} \mathrm{C}$ com $90 \% \mathrm{UR}+/-5 \%$, pelo período de 12 dias, sendo avaliado a cada 2 dias.

Oexperimento foi dividido em dois grupos: o controle (não destrutivo) e o grupo parcela (destrutivo).
Para o grupo controle foram utilizados 10 embalagens por tratamento ( 25 frutos/embalagem), analisados a aparência, o tempo de vida útil e a incidência de doenças. Para o grupo parcela foi utilizado uma embalagem por parcela, com total de 3 embalagens por tratamento por dia de avaliação, e analisados quanto ao teor de sólidos solúveis, acidez titulável, teor de vitamina $\mathrm{C}$ e firmeza.

A avaliação da aparência foi feita de acordo com as normas do Instituto Adolfo Lutz (1985). Ao produto em ótimas condições sensoriais e de comercialização se atribuiu a nota 3 , ao produto em condições sensoriais e de comercialização aceitáveis se atribuiu nota 2 , e ao produto em condições sensoriais e de comercialização inadequadas se atribuiu a nota 1 . O tempo de vida útil dos frutos foi estabelecido em função do número de dias em que os frutos permaneceram em condições aceitáveis para comercialização (nota 2 de aparência). As avaliações sensórias foram feitas por 22 provadores não treinados

A incidência de doenças foi determinada em percentagem, considerando o ponto inicial com $100 \%$ das embalagens com frutos sadios.

O teor de sólidos solúveis, acidez titulável e vitamina $\mathrm{C}$, foram feitas de acordo com as normas do Instituto Adolfo Lutz (1985) e a firmeza foi determinada em (g/f), medida nos frutos inteiros, utilizando-se o Texturômetro Stevens - LFRA Texture Analyser, com ponta de prova TA 9/1000, velocidade de penetração de $2,0 \mathrm{~mm} \mathrm{seg}^{-1}$ e profundidade de $15 \mathrm{~mm}$.

O delineamento estatístico empregado foi o inteiramente casualizado, com 5 tratamentos e 3 repetições para o grupo parcela e 10 repetições para o grupo controle.

Para comparação entre as médias, foi utilizado o teste de Tukey ao nível de $5 \%$ de probabilidade (GOMES, 1987). 


\section{Resultados e Discussão}

Observando os dados da Tabela 1, pode-se dizer que as diferentes atmosferas modificadas não tiveram influência sobre a aparência dos frutos, já que em todos os dias de avaliação,os frutos dos tratamentos não apresentaram diferenças estatísticas entre si.

Tabela 1. Variação média da aparência* dos morangos armazenados em atmosfera modificada nos diferentes tratamentos.

\begin{tabular}{lcccccccc}
\hline & \multicolumn{7}{c}{ Dias de Armazenamento } & CV \\
\hline Tratamentos & 0 & 2 & 4 & 6 & 8 & 10 & 12 & $(\%)$ \\
\hline Testemunha & $3,0 \mathrm{aA}$ & $3,0 \mathrm{aA}$ & $3,0 \mathrm{aA}$ & $3,0 \mathrm{aA}$ & $2,9 \mathrm{aA}$ & $2,9 \mathrm{aA}$ & $2,4 \mathrm{aB}$ & 9,78 \\
Vácuo parcial & $3,0 \mathrm{aA}$ & $2,8 \mathrm{aA}$ & $2,8 \mathrm{aA}$ & $2,8 \mathrm{aA}$ & $2,6 \mathrm{aAB}$ & $2,6 \mathrm{aAB}$ & $2,0 \mathrm{aB}$ & 17,83 \\
$5 \% \mathrm{O}_{2} / 1 \% \mathrm{CO}_{2}$ & $3,0 \mathrm{aA}$ & $3,0 \mathrm{aA}$ & $2,8 \mathrm{aA}$ & $2,8 \mathrm{aA}$ & $2,8 \mathrm{aA}$ & $2,4 \mathrm{aAB}$ & $2,4 \mathrm{aB}$ & 18,80 \\
$5 \% \mathrm{O}_{2} / 3 \% \mathrm{CO}_{2}$ & $3,0 \mathrm{aA}$ & $3,0 \mathrm{aA}$ & $2,9 \mathrm{aAB}$ & $2,9 \mathrm{aAB}$ & $2,6 \mathrm{aAB}$ & $2,6 \mathrm{aAB}$ & $2,0 \mathrm{aB}$ & 15,30 \\
$5 \% \mathrm{O}_{2} / 5 \% \mathrm{CO}_{2}$ & $3,0 \mathrm{aA}$ & $3,0 \mathrm{aA}$ & $2,7 \mathrm{aAB}$ & $2,7 \mathrm{aAB}$ & $2,7 \mathrm{aAB}$ & $2,5 \mathrm{aAB}$ & $2,2 \mathrm{aB}$ & 16,68 \\
\multicolumn{1}{c}{$\mathrm{CV} \%$} & 0,00 & 6,46 & 13,09 & 13,09 & 16,80 & 18,67 & 21,04 &
\end{tabular}

Médias seguidas pela mesma letra minúscula na coluna e maiúscula na linha, não diferem significativamente entre si, a $5 \%$ de probabilidade, pelo teste de Tukey.

*Nota 3 (bom), 2 (razoável) e 1 (ruim ou impróprio).

Do ponto inicial até o $10^{\circ}$ dia de armazenamento, os frutos de todos os tratamentos, principalmente da testemunha, apresentaram boas condições de comercialização. Porém, no $12^{\circ}$ dia, observou-se que a maioria dos frutos sofreu queda acentuada em sua aparência, obtendo notas que variaram entre 2,0 e 2,4 , sendo, portanto considerados produtos razoáveis para a sua comercialização.

Levando-se em consideração a aparência do morango armazenado em atmosfera modificada e sob refrigeração, Collins e Perkins-Veazie (1993) observaram que a queda na qualidade visual dos frutos durante o armazenamento foi pequena em comparação com os frutos armazenados em ambiente atmosférico normal. Os mesmos autores verificaram que a refrigeração foi vital para assegurar a manutenção da aparência.

Na Tabela 2, pode-se verificar a variação média da vida útil do morango para comercialização, no qual se pode verificar diferença estatística significativa entre os frutos da testemunha e dos armazenados em vácuo parcial. Esses resultados são discordantes dos relatados por Chitarra e Chitarra (1990), onde citam que o período máximo de vida pós-colheita para o morango (10 dias) só é atingido se os frutos forem mantidos em temperaturas de $0-5^{\circ} \mathrm{C}, 10 \% \mathrm{O}_{2}$ e 15 -
$20 \% \mathrm{CO}_{2}$. Calegaro et al (2002) verificaram um período máximo de 7 dias de conservação de morangos cv. Oso Grande associados à atmosfera modificada.

No entanto resultados mais próximos dos obtidos neste experimento foram encontrados por Scalon et al (1996), que verificaram 14 dias pós-colheita para morangos armazenados em PVC a $4^{\circ} \mathrm{C}$. Ainda, Brackmann et al (2001) verificaram que a conservação de morangos cv. Oso Grande a $0^{\circ} \mathrm{C}$ e pressão parcial de $20 \%$ de $\mathrm{CO}_{2}$ proporcionaram conservação, sem perda de qualidade, por até 20 dias.

Com relação a variação média percentual da incidência de doenças, verifica-se pela Tabela 3 que os frutos mantiveram-se íntegros até o $8^{\circ}$ dia de armazenamento. A partir do $10^{\circ}$ dia, detectou-se a presença de doenças nos morangos causada principalmente por Botrytis cineria, sendo que ao final do $12^{\circ}$ dia os frutos do armazenamento a vácuo apresentaram a menor incidência de doenças. Entretanto, as percentagens de doenças encontradas nos frutos dos diferentes tratamentos foram semelhantes e relativamente baixas, quando comparadas a outros trabalhos onde as frutas foram armazenadas apenas sob refrigeração, como descritos por Silva (2002). 
Tabela 2. Variação média da vida útil (dias) para comercialização dos morangos armazenados em atmosfera modificada nos diferentes tratamentos.

Tratamentos

Testemunha

Vácuo parcial

$5 \% \mathrm{O}_{2} / 1 \% \mathrm{CO}_{2}$

$5 \% \mathrm{O}_{2} / 3 \% \mathrm{CO}_{2}$

$5 \% \mathrm{O}_{2} / 5 \% \mathrm{CO}_{2}$

$\mathrm{CV} \%$
Vida útil para comercialização

(Dia)

$16,3 \mathrm{a}$

$11,8 \mathrm{~b}$

$15,0 \mathrm{ab}$

$12,7 \mathrm{ab}$

$12,3 \mathrm{ab}$

23,09

Médias seguidas pela mesma letra não diferem significativamente entre si, a $5 \%$ de probabilidade, pelo teste de Tukey.

Tabela 3. Variação média percentual da incidência de doenças dos morangos armazenados em atmosfera modificada nos diferentes tratamentos.

\begin{tabular}{llllllll}
\hline & \multicolumn{7}{c}{ Dias de Armazenamento } \\
\hline Tratamentos & 0 & 2 & 4 & 6 & 8 & 10 & 12 \\
Testemunha & 0 & 0 & 0 & 0 & 0 & 20 & 30 \\
Vácuo parcial & 0 & 0 & 0 & 0 & 0 & 0 & 20 \\
$5 \% \mathrm{O}_{2} / 1 \% \mathrm{CO}_{2}$ & 0 & 0 & 0 & 0 & 0 & 10 & 30 \\
$5 \% \mathrm{O}_{2} / 3 \% \mathrm{CO}_{2}$ & 0 & 0 & 0 & 0 & 0 & 10 & 30 \\
$5 \% \mathrm{O}_{2} / 5 \% \mathrm{CO}_{2}$ & 0 & 0 & 0 & 0 & 0 & 20 & 30 \\
\hline
\end{tabular}

$\mathrm{O}$ aumento da concentração de $\mathrm{CO}_{2}$ no armazenamento de produtos vegetais, entre eles o morango, reduz de forma indireta a incidência de patógenos, sendo esse controle variável de acordo com a espécie do produto e o patógeno envolvido. Segundo Flores-Castillano (1998) a deterioração e podridões aumentam com o período de conservação. Entretanto, a atmosfera modificada gerada pela fruta no interior de filmes poliméricos, pode reduzir esses problemas.

Os resultados obtidos neste experimento são concordantes com os obtidos por Hertog et al (1999) e Van der Steen et al (2002), onde se verificou que as elevadas concentrações de $\mathrm{CO}_{2}$ no armazenamento de morangos foram eficientes na redução de perdas causadas por patógenos, principalmente por Botrytis cineria.

Os teores de sólidos apresentados pelos morangos neste experimento encontram-se na Tabela 4. Pelos resultados pode-se dizer que durante todo o período de armazenamento não ocorreram variações significativas nos frutos dos diferentes tratamentos. Esses resultados se assemelham aos encontrados por Collins e Perkins-Veazie (1993), os quais verificaram que os teores de sólidos solúveis em morangos não variaram significativamente em todo o seu armazenamento quando armazenados em diferentes temperaturas.

Apesar de não ter ocorrido diferença significativa nos teores de sólidos solúveis nos frutos de todos os tratamentos, verifica-se tendência de queda nestes teores principalmente na fase final do experimento. Estes resultados são concordantes com os encontrados por Gil et al (1997), que trabalhando com morangos armazenados com atmosferas modificadas sob diferentes concentrações de $\mathrm{CO}_{2}$, verificaram que os teores de sólidos solúveis diminuíram depois de 5 e 10 dias de armazenamento a $5^{\circ} \mathrm{C}$. Os valores de sólidos solúveis encontrados para os morangos deste experimento são superiores aos encontrados por Berbari et al (1994). 
Tabela 4. Variação média de sólidos solúveis ( $\left.{ }^{\circ} B r i x\right)$ dos morangos armazenados em atmosfera modificada nos diferentes tratamentos.

\begin{tabular}{|c|c|c|c|c|c|c|c|c|}
\hline \multirow[b]{2}{*}{ Tratamentos } & \multicolumn{7}{|c|}{ Dias de Armazenamento } & \multirow{2}{*}{$\begin{array}{l}\mathbf{C V} \\
(\%)\end{array}$} \\
\hline & 0 & 2 & 4 & 6 & 8 & 10 & 12 & \\
\hline Testemunha & $7,60 \mathrm{aA}$ & $7,70 \mathrm{aA}$ & $7,60 \mathrm{aA}$ & $7,30 \mathrm{aA}$ & $7,30 \mathrm{aA}$ & $7,30 \mathrm{aA}$ & $7,30 \mathrm{aA}$ & 6,09 \\
\hline Vácuo parcial & $7,60 \mathrm{aA}$ & $7,50 \mathrm{aA}$ & $7,30 \mathrm{aA}$ & $7,30 \mathrm{aA}$ & $7,20 \mathrm{aA}$ & $7,30 \mathrm{aA}$ & $7,70 \mathrm{aA}$ & 5,54 \\
\hline $5 \% \mathrm{O}_{2} / 1 \% \mathrm{CO}_{2}$ & $7,60 \mathrm{aA}$ & $7,40 \mathrm{aA}$ & $7,20 \mathrm{aA}$ & $7,50 \mathrm{aA}$ & $7,50 \mathrm{aA}$ & $7,20 \mathrm{aA}$ & $7,60 \mathrm{aA}$ & 4,45 \\
\hline $5 \% \mathrm{O}_{2} / 3 \% \mathrm{CO}_{2}$ & $7,60 \mathrm{aA}$ & $7,50 \mathrm{aA}$ & $7,60 \mathrm{aA}$ & $7,40 \mathrm{aA}$ & $7,10 \mathrm{aA}$ & $7,20 \mathrm{aA}$ & $7,10 \mathrm{aA}$ & 5,48 \\
\hline $5 \% \mathrm{O}_{2} / 5 \% \mathrm{CO}_{2}$ & $7,60 \mathrm{aA}$ & $7,40 \mathrm{aA}$ & $7,30 \mathrm{aA}$ & $7,40 \mathrm{aA}$ & $7,60 \mathrm{aA}$ & $7,20 \mathrm{aA}$ & $7,10 \mathrm{aA}$ & 5,98 \\
\hline $\mathrm{CV} \%$ & 0,00 & 5,90 & 3,77 & 5,38 & 4,60 & 7,25 & 5,74 & \\
\hline
\end{tabular}

Médias seguidas pela mesma letra minúscula na coluna e maiúscula na linha, não diferem significativamente entre si, a $5 \%$ de probabilidade, pelo teste de Tukey.

Os resultados da avaliação da acidez titulável dos frutos apresentados na Tabela 5 concordam com as citações de Chitarra e Chitarra (1990), Sigrist (1992) e Scalon (1996). Todos citam que os teores de acidez titulável dos frutos tendem a cair durante o seu armazenamento, fato esse verificado para o morango neste experimento.

Tabela 5. Variação média de acidez titulável (g de ácido cítrico/100g de tecido fresco) dos morangos armazenados em atmosfera modificada nos diferentes tratamentos.

\begin{tabular}{lccccccrc}
\hline & & \multicolumn{4}{c}{ Dias de Armazenamento } & \multicolumn{2}{c}{ CV } \\
Tratamentos & 0 & 2 & 4 & 6 & 8 & 10 & 12 & $(\%)$ \\
\hline Testemunha & $0,81 \mathrm{aAB}$ & $0,76 \mathrm{aBC}$ & $0,85 \mathrm{aA}$ & $0,80 \mathrm{aAB}$ & $0,81 \mathrm{aAB}$ & $0,72 \mathrm{aC}$ & $0,78 \mathrm{aABC}$ & 3,72 \\
Vácuo parcial & $0,81 \mathrm{aA}$ & $0,75 \mathrm{aAB}$ & $0,72 \mathrm{aAB}$ & $0,78 \mathrm{aAB}$ & $0,74 \mathrm{aAB}$ & $0,71 \mathrm{aB}$ & $0,68 \mathrm{bAB}$ & 6,62 \\
$5 \% \mathrm{O}_{2} / 1 \% \mathrm{CO}_{2}$ & $0,81 \mathrm{aAB}$ & $0,83 \mathrm{aA}$ & $0,77 \mathrm{aAB}$ & $0,82 \mathrm{aAB}$ & $0,74 \mathrm{aAB}$ & $0,72 \mathrm{aB}$ & $0,73 \mathrm{abAB}$ & 5,2 \\
$5 \% \mathrm{O}_{2} / 3 \% \mathrm{CO}_{2}$ & $0,81 \mathrm{aA}$ & $0,77 \mathrm{aABC}$ & $0,78 \mathrm{aABC}$ & $0,79 \mathrm{aAB}$ & $0,74 \mathrm{aBC}$ & $0,71 \mathrm{aC}$ & $0,72 \mathrm{abC}$ & 3,24 \\
$5 \% \mathrm{O}_{2} / 5 \% \mathrm{CO}_{2}$ & $0,81 \mathrm{aA}$ & $0,85 \mathrm{aA}$ & $0,81 \mathrm{aA}$ & $0,80 \mathrm{aA}$ & $0,72 \mathrm{aA}$ & $0,67 \mathrm{aA}$ & $0,72 \mathrm{abA}$ & 11,95 \\
\multicolumn{1}{c}{$\mathrm{CV} \%$} & 0,00 & 4,88 & 6,43 & 4,99 & 5,14 & 3,58 & 3,8 & \\
\hline
\end{tabular}

Médias seguidas pela mesma letra minúscula na coluna e maiúscula na linha, não diferem significativamente entre si, a $5 \%$ de probabilidade, pelo teste de Tukey.

Para os frutos climatéricos a diminuição da acidez durante o período de armazenamento é vinculada principalmente ao processo respiratório e a conversão dos ácidos em açúcares no qual caracteriza o seu amadurecimento. Neste experimento a diminuição no teor de acidez titulável nos morangos deve-se provavelmente, a sua utilização no processo respiratório e na sua degradação durante o armazenamento, e não a sua utilização na conversão de açúcares. Fato este verificado pela pequena variação no teor de sólidos solúveis encontrado.
Os valores médios de vitamina $\mathrm{C}$ dos frutos de morango encontrados na Tabela 6 nos mostram que ao longo do período de armazenamento ocorreram diminuição dos teores para os frutos de todos os tratamentos testados. Esses resultados são condizentes com os observados por ParaskevopoulouParoussi e Vassilakakis (1995), Nunes et al (1998) e Calegaro et al (2002). Segundo Islan et al (1993) esta redução se dá devido a alta atividade pós-colheita da enzima ácido ascórbico oxidase. Para Gayon (1972) a diminuição dos teores de vitamina $\mathrm{C}$ na pós-colheita pode ser devido à menor capacidade dos vegetais de sintetizar esse ácido durante esse período. 
Vieites, R. L. et al.

Tabela 6. Variação média do teor de vitamina C (mg/100g de tecido fresco) dos morangos armazenados em atmosfera modificada nos diferentes tratamentos.

\begin{tabular}{|c|c|c|c|c|c|c|c|c|}
\hline \multirow[b]{2}{*}{ Tratamentos } & \multicolumn{7}{|c|}{ Dias de Armazenamento } & \multirow{2}{*}{$\begin{array}{l}\text { CV } \\
(\%)\end{array}$} \\
\hline & 0 & 2 & 4 & 6 & $8 *$ & 10 & $12 *$ & \\
\hline Testemunha & $44,6 \mathrm{aB}$ & $49,4 \mathrm{aA}$ & $47,9 \mathrm{aAB}$ & $44,8 \mathrm{aB}$ & $44,2 \mathrm{aB}$ & $39,7 \mathrm{aCD}$ & $30,2 \mathrm{aD}$ & 3,57 \\
\hline Vácuo parcial & $44,6 \mathrm{aA}$ & $45,0 \mathrm{abA}$ & $42,6 \mathrm{bB}$ & $44,3 \mathrm{aA}$ & $43,7 \mathrm{aB}$ & $38,6 \mathrm{aC}$ & $32,1 \mathrm{aD}$ & 6,83 \\
\hline $5 \% \mathrm{O}_{2} / 1 \% \mathrm{CO}_{2}$ & $44,6 \mathrm{aB}$ & $46,5 \mathrm{abA}$ & $45,6 \mathrm{abA}$ & $45,8 \mathrm{aA}$ & $44,0 \mathrm{aB}$ & $38,8 \mathrm{aC}$ & $33,0 \mathrm{aD}$ & 8,81 \\
\hline $5 \% \mathrm{O}_{2} / 3 \% \mathrm{CO}_{2}$ & $44,6 \mathrm{aB}$ & $43,3 \mathrm{abB}$ & $45,9 \mathrm{abAB}$ & $49,1 \mathrm{aA}$ & $44,2 \mathrm{aB}$ & $37,6 \mathrm{aC}$ & $32,7 \mathrm{aD}$ & 3,39 \\
\hline $5 \% \mathrm{O}_{2} / 5 \% \mathrm{CO}_{2}$ & $44,6 \mathrm{aA}$ & $41,7 \mathrm{bB}$ & $46,3 \mathrm{abA}$ & $45,3 \mathrm{aA}$ & $43,8 \mathrm{aB}$ & $36,9 \mathrm{aC}$ & $32,9 \mathrm{aD}$ & 5,49 \\
\hline $\mathrm{CV} \%$ & 0,00 & 5,57 & 4,09 & 8,83 & 6,62 & 5,54 & 3,22 & \\
\hline
\end{tabular}

Médias seguidas pela mesma letra minúscula na coluna e maiúscula na linha, não diferem significativamente entre si,a $5 \%$ de probabilidade, pelo teste de Tukey.

Com os resultados apresentados neste experimento, verificou-se que a atmosfera modificada não influenciou nos teores de vitamina $\mathrm{C}$ dos frutos de todos os tratamentos estudados.

Na Tabela 7 pode-se avaliar a variação média da firmeza dos frutos submetidos às diferentes atmosferas modificadas. De acordo com Santos
(1999), a firmeza da polpa e a resistência da epiderme são características de extrema importância, especialmente para as cultivares destinadas à produção de fruto para o consumo in natura, pois além de permitirem melhor manuseio e transporte, possibilitam a conservação das qualidades sensoriais por mais tempo, fato este verificado neste experimento.

Tabela 7. Variação média da firmeza $\left(\mathrm{gf} / \mathrm{cm}^{-3}\right)$ dos morangos armazenados em atmosfera modificada nos diferentes tratamentos.

\begin{tabular}{|c|c|c|c|c|c|c|c|c|}
\hline \multirow[b]{2}{*}{ Tratamentos } & \multicolumn{7}{|c|}{ Dias de Armazenamento } & \multirow{2}{*}{$\begin{array}{l}\mathbf{C V} \\
(\%)\end{array}$} \\
\hline & 0 & 2 & 4 & 6 & 8 & 10 & 12 & \\
\hline Testemunha & $52,3 \mathrm{aB}$ & $82,7 \mathrm{aAB}$ & $102,4 \mathrm{abA}$ & 83,3 abAB & $98,7 \mathrm{aAB}$ & $87,4 \mathrm{aAB}$ & $122,5 \mathrm{aA}$ & 18,77 \\
\hline Vácuo parcial & $52,3 \mathrm{aC}$ & $58,8 \mathrm{abBC}$ & $128,0 \mathrm{aA}$ & $93,2 \mathrm{aAB}$ & $101,5 \mathrm{aA}$ & $97,7 \mathrm{aA}$ & $109,8 \mathrm{aA}$ & 13,90 \\
\hline $5 \% \mathrm{O}_{2} / 1 \% \mathrm{CO}_{2}$ & $52,3 \mathrm{aA}$ & $55,2 \mathrm{bA}$ & $66,7 \mathrm{bA}$ & $65,3 \mathrm{bA}$ & $90,7 \mathrm{aA}$ & $82,7 \mathrm{aA}$ & $88,7 \mathrm{aA}$ & 29,61 \\
\hline $5 \% \mathrm{O}_{2} / 3 \% \mathrm{CO}_{2}$ & $52,3 \mathrm{aC}$ & 59,4 abBC & 89,9 abAB & $68,5 \mathrm{bABC}$ & $77,9 \mathrm{aABC}$ & $77,5 \mathrm{aABC}$ & $103,4 \mathrm{aA}$ & 17,72 \\
\hline $5 \% \mathrm{O}_{2} / 5 \% \mathrm{CO}_{2}$ & $52,3 \mathrm{aC}$ & $61,9 \mathrm{abBC}$ & 88,9 abAB & $63,2 \mathrm{bBC}$ & $69,9 \mathrm{aBC}$ & $70,9 \mathrm{aBC}$ & $107,2 \mathrm{aA}$ & 14,73 \\
\hline $\mathrm{CV} \%$ & 0,00 & 15,19 & 18,88 & 10,14 & 17,26 & 14,86 & 26,11 & \\
\hline
\end{tabular}

Médias seguidas pela mesma letra minúscula na coluna e maiúscula na linha, não diferem significativamente entre si, a $5 \%$ de probabilidade, pelo teste de Tukey.

Frutos de todos os tratamentos apresentaram acréscimos nos valores de firmeza durante o armazenamento, correspondendo em média duas vezes a firmeza inicial. Apesar de não ter sido quantificado a concentração final de $\mathrm{O}_{2}$ e $\mathrm{CO}_{2}$ dentro das embalagens ao final do armazenamento, é sabido que, no decorrer do período de armazenamento de morangos em atmosferas modificadas, há um acúmulo de $\mathrm{CO}_{2}$, com queda na concentração de $\mathrm{O}_{2}$, observações estas concordantes com (KAYS, 1991, NUNES et al, $1998 \mathrm{e}$ VAN DER STEEN et al, 2002).
Estudos realizados com morangos verificaram que quando a fruta é armazenada com altas concentrações de $\mathrm{CO}_{2}$, mantém a sua firmeza por mais tempo (HARRIS e HARVEY, 1973, 1978, MITCHELL, 1992), dados esses concordantes com os encontrados neste experimento. Li e Kader (1989) observaram que morangos armazenados por até 4 dias com $20 \%$ de CO2, mantiveram sua firmeza por maior período de tempo do que as frutas tratadas durante apenas 2 dias. 
Harker et al (2000) em experimentos com morangos armazenados em elevadas concentrações de $\mathrm{CO}_{2}$, encontraram resultados semelhantes a este experimento. Eles verificaram aumento de firmeza do morango em função da elevação da concentração de $\mathrm{CO}_{2}$ no ambiente de armazenamento, concordando também com Holcroft e Kader (1999). Esses dois autores relatam que a atmosfera com elevada concentração de $\mathrm{CO}_{2}$ foi eficiente no controle e retenção da firmeza de morangos armazenados durante 10 dias a $5^{\circ} \mathrm{C}$, enquanto que as frutas armazenadas em condições atmosféricas normais amoleceram muito mais rápido nas mesmas condições de temperatura.

\section{Conclusões}

Nas condições em que foi desenvolvido este experimento pode-se concluir que não houve diferença com os demais tratamentos exceto o tratamento com vácuo parcial, mesmo que a elevada concentração de $\mathrm{CO}_{2}$ tenha sido importante para a elevação da firmeza do morango. As atmosferas modificadas ativas estudadas não apresentaram diferenças em relação a testemunha até a modificada passiva na manutenção da qualidade e conservação dos frutos.

\section{Agradecimentos}

Agradecemos a FAPESP pelo financiamento desta pesquisa.

\section{Referências}

BERBARI, S.A.G.; NOGUEIRA, J.N.; PASSOS, F.A. Determinações das características físicas, químicas e organolépticas de novas variedades de morango para congelamento. Boletim BCTA, v.28, n.1, p.18-24, 1994.

BRACKMANN, A .et al. Armazenamento de morangos cv. Oso Grande (Fragaria Ananassa L.) sob elevadas pressões parciais de $\mathrm{CO}_{2}$. Revista Brasileira de Agrociência, v.7, n.1, p.10-14, 2001.
CALEGARO, J.J.; PEZZI, E.; BENDER, R.J. Utilização de atmosfera modificada na conservação de morangos em pós-colheita. Pesquisa Agropecuária Brasileira, Brasília, v.37, n.8, 2002.

CHITARRA, M.I.F.; CHITARRA, A .B. Pós-colheita de frutos e hortaliças: fisiologia e manuseio. Lavras: ESAL: FAEPE, 1990.320p.

CIA, P. Efeito da atmosfera modificada no controle de podridões pós-colheita e na qualidade de caqui cv. Fuyu. 2002. 122f. Dissertação (Mestrado em Agronomia/ Horticultura) - Faculdade de Ciências Agronômicas, Universidade Estadual Paulista, Botucatu, 2002.

COLLINS, J.K.; PERKINS-VEAZIE, P. Postharvest changes in strawberry fruit stored under simulated retail display conditions. Journal of Food Quality, Trumbull, v.16, p.13343, 1993.

FLORES-CANTILLANO, F. Estudio del efecto de las atmósferas modificadas durante el almacenamiento y comercialización de algumas frutas e hortalizas. 1998. 276 f. Tesis Doctoral-Universidad Politécnica de Valencia, Valencia España. 1998.

GAYON, P.R. Plant Phenolics. Oliver and Boyd, Edingurg, 1972.

GIL, M.I.; HOLCROFT, D.M.; KADER, A .A . Changes in strawberry anthoctanins and other polyphenols in response to carben dioxide treatments. Journal Agricultural Food Chemistry, Washington, v. 45, p. 166267, 1997.

GOMES, F.P. Curso de Estatística Experimental. 12. ed. Piracicaba: Nobel, 1987. 467 p.

HARKER, R.F. et al. Physical and mechanical changes in strawberries after high carbon dioxide treatments. Postharvest Biology and Technology, Amsterdan, v. 19, p. 139-46, 2000.

HARRIS, C.M.; HARVEY, J.M. Quality and decay of California strawberries stored in $\mathrm{CO}_{2}$ enriched atmospheres. Plant Disease Reporter, Washington, v. 57, p. 44-46, 1973.

HERTOG, M.L.A.T.M. et al. Predicting keeping quality of strawberries (cv. 'Elsanta') packed under modified atmospheres: na integrated model approach. Postharvest Biology and Technology, Amsterdan, v. 15, n. 1, p. 1-12, 1999.

HOLCROFT, D.M.; KARDER, A.A. Controlled atmosphere in duced changes in $\mathrm{pH}$ and organic acid metabolism may affect color of stored strawberry fruit. Postharvest Biology and Technology, Amsterdan, v.17, p. 19-32, 1999. 
INSTITUTO ADOLFO LUTZ. Métodos fisicos e químicos para análise de alimentos. 3. ed. São Paulo: Instituto Adolfo Lutz, 1985. 533 p.

ISLAN, M.N.; COLON, T.; VARGAS, T. Effect of prolonged solar exposure on the vitamin $\mathrm{C}$ contents of tropical fruits. Food Chemistry, Linton Road Barking, v. 48, p. 75-8, 1993.

KADER, A.A.; ZAGORY, D.; KERBEL, E.L. Modified atmosphere packaging of fruits and vegetables. Critical Reviews in Food Science and Nutrition. West Palm Beach, v. 28, n. 1, p. 1-30, 1989.

KADER, A.A. Postharvest Technology of horticultural crops. 2 ed. Oakland: Division of agricultural and Natural Resources, University of California, 1992.296 p.

KAYS, S.J. . Postharvest physiology of perishable plant products, Van Nostrand-Reinhold: New York. 1991.

KLUGE, R.A. et al. Distúrbios Fisiológicos em Frutos. Piracicaba: FEALQ, 2001.58 p.

LI,C.; KADER,A.A. Residual affects of controlled atmosphere on postharvest physiology and quality of strawberries. Journal American Horticultural Science, Mount, v. 1144, n. 4, p. 629-634. 1989.

MITCHELL, F.G. postharvest handling system: small fruits (table grapes, strawberries, kiwi-fruit). In: KARDER, A.A. (ed.). Postharvest Technology of horticultural crops. 2 ed. Univ. of California Publ. P. 223-231. 1992.

NUNES, M.C.N. et al. Controlling temperature and watwe loss to maintain ascorbic acid levels in strawberries during postharvest handling. Journal of Food Science, Chicago, v. 63 , n. 6, p. $1033-36,1998$.
PARASKEVOPOULOU-PAROUSSI, G.; VASSILAKAKIS, M. Effects of temperature, duration of cold storage and packaging on postharvest quality of strawberry fruit. Acta Hortic., n.379. p.337-344, 1995.

SANTOS, A.M. Melhoramento genético do morangueiro. Informe Agropecuário, Belo Horizonte, v. 20, n. 198, p. 24$9,1999$.

SARANTÓPOULOS, C.I.G.L. et al. Embalagens com atmosfera modificada. Campinas: ITAL, 1996. $114 \mathrm{p}$.

SCALON, S.P.Q. et al Conservação de morangos (Fragaria Ananassa Duch) cv. Sequóia em atmosfera modificada. Revista Brasileira de Fruticultura, Cruz das Almas, v. 18, n. 3, p. 431-36, 1996.

SIGRIST, J.M.M. Transformações bioquímicas. In: BLEINROTH, E.W. et al. Tecnologia de pós-colheita de frutas tropicais. 2 ed. Campinas: ITAL, 1992. p. 33-40.

SILVA, A.P.Qualidade e conservação pós-colheita do morango, tratado com cloreto de cálcio em pré-colheita. 2002. 132p. (Tese de Doutorado em Agronomia/ Horticultura) Faculdade de Ciências Agronômicas, UNESP, Botucatu, 2002.

SILVA, C.S. Qualidade e conservação do morango tratado em pós-colheita com cloreto de cálcio e do armazenamento em atmosfera modificada ativa. 2004. 96p. (Tese de Doutorado em Agronomia/Horticultura) Faculdade de Ciências Agronômicas, UNESP, Botucatu, 2004.

VAN DER STEEN, C.; JACXSENS, L.; DEVLIEGHERE, F. Combining high oxygen atmospheres with low oxygen modified atmosphere packaging to improve the keeping quality of strawberries and raspberries. Postharvest Biology and Technology, Amsterdan, v. 26, n. 1, p. 49-58, 2002. 